\title{
Ensayos no destructivos y rehabilitación de elevadores de vehículos ferroviarios
}

\section{Non-destructive testing and rehabilitation of railway vehicle elevators}

Luis Fernando Buenaño Moyano. ${ }^{1}$, Edison Marcelo Castillo Cárdenas. ${ }^{2}$, Bolívar Alejandro Cuaical Angulo. ${ }^{3} \&$ Celin Abad Padilla Padilla. ${ }^{4}$

\begin{abstract}
.
DOI: https://doi.org/10.33262/cienciadigital.v3i1.295

The analysis of the operation and subsequent rehabilitation of the electromechanical elevators of Ferrocarriles del Ecuador Empresa Pública (FEPP) has been carried out. To check the status of each of the elevators, a matrix of valuation and non-destructive tests (NDT) was used. The evaluation matrix identified each of the anomalies and faults that existed in the elements of the elevators. The NDTs were used to identify discontinuities in the welding used. The digitalization of the elevators and the subsequent stress analysis in Ansys were also carried out in order to determine the load to which each of the elevators may be subjected, concluding that to lift 55.65 tons, a minimum of 4 elevators must be used to ensure a safety factor of 1.13. Finally, the elevators were rehabilitated through maintenance, and the replacement or implementation of each of the elements previously inspected. After the rehabilitation, a field test was carried out by raising a 17-ton panoramic car, and the functionality of the four elevators that will be used in the maintenance workshops of FEEP was demonstrated.
\end{abstract}

\footnotetext{
1 Escuela Superior Politécnica de Chimborazo, Facultad de Mecánica, Chimborazo, Ecuador, lfbuenanio@espoch.edu.ec

2 Escuela Superior Politécnica de Chimborazo, Facultad de Mecánica, Chimborazo, Ecuador, edison.castillo@espoch.edu.ec

3 Escuela Superior Politécnica de Chimborazo, Facultad de Mecánica, Chimborazo, Ecuador, bcuaical@espoch.edu.ec

4 Escuela Superior Politécnica de Chimborazo, Facultad de Mecánica, Chimborazo, Ecuador, c_padilla@espoch.edu.ec
} 
Keywords: Analysis of Efforts, Computer Aided Design, Electromechanical Elevators, Non Destructive Tests, Locomotives.

\section{Resumen.}

Se ha realizado el análisis del funcionamiento y la posterior rehabilitación de los elevadores electromecánicos de Ferrocarriles del Ecuador Empresa Pública (FEPP). Para comprobar el estado de cada uno de los elevadores se utilizó una matriz de valoración y ensayos no destructivos (END). La matriz de valoración identificó cada una de las anomalías y fallas que existían en los elementos de los elevadores. Los END se utilizaron para la identificación de discontinuidades en la soldadura empleada. Se realizó también la digitalización de los elevadores y el posterior análisis de esfuerzos en Ansys con el fin de determinar la carga a la cual pueden estar sometidos cada uno de los elevadores concluyendo que para levantar $55.65 \mathrm{t}$ se debe utilizar un mínimo de 4 elevadores para asegurar un factor de seguridad de 1.13. Finalmente se procedió a la rehabilitación de los elevadores mediante el mantenimiento, y la sustitución o la implementación de cada uno de los elementos inspeccionados previamente. Luego de la rehabilitación se realizó una prueba de campo elevando un vagón panorámico de $17 \mathrm{t}$, y se evidenció el buen funcionamiento de los cuatro elevadores que se usarán en los talleres de mantenimiento de FEEP.

Palabras claves: Análisis de Esfuerzos, Diseño Asistido por Computadora, Elevadores Electromecánicos, Ensayos no Destructivos, Locomotoras.

\section{Introducción.}

En el Ecuador Ferrocarriles del Ecuador Empresa Pública (FEEP), está encargada de la administración del sistema ferroviario del país, el cual es uno de los puntos fuertes a nivel turístico a nivel local e internacional, las actividades de mantenimiento en los sistemas ferroviarios, son indispensables para que este tipo de industria pueda desarrollarse adecuadamente, y siendo FEEP quien asume también las labores de mantenimiento de sus activos esta debe procurar cumplir con todo los tipos de servicio de mantenimiento.

Con el propósito del montaje y desmontaje de los vagones y sus partes, y del mantenimiento de locomotoras es necesario que se requiera la suspensión sobre el suelo mediante elevadores mecánicos adecuados para lograr este cometido, tomando en cuenta que se debe contar con las condiciones de confiablidad y seguridad necesarias para poder realizarlo en el taller de mantenimiento sin riesgo y durante el tiempo que la tarea de mantenimiento requiera.

De esta manera surge entonces la necesidad de contar con la utilidad absoluta de los elevadores electromecánicos con los que el taller de mantenimiento de FEEP matriz Riobamba cuenta. Los elevadores que se posee en la empresa son de tipo electromecánico, que en un principio fueron mecánicos, utilizándose manivelas para su funcionamiento y que 
actualmente cuentan con unos motores eléctricos que ayudan a elevar y descender a los vagones; facilitando el trabajo de tal forma que ayuda en la optimización de tiempos muertos al momento de su utilización.

Sin embargo, debido al tiempo de uso y la falta de mantenimiento, es necesario el diagnóstico para su rehabilitación y repotenciación haciéndose pertinente además que para un trabajo adecuado y responsable se aporte con los parámetros técnicos de funcionamiento, en este caso el factor de seguridad, la carga máxima de operación y el que se diagnostique técnicamente el estado de los elevadores para que la rehabilitación sea eficaz.

El presente trabajo tiene como objetivo principal el diagnosticar y rehabilitar los elevadores electromecánicos para vehículos ferroviarios de la empresa FEEP, utilizando las técnicas de ingeniería más adecuadas, en este caso el análisis de esfuerzos mediante CAD y la aplicación de ensayos no destructivos a fin de determinar su funcionalidad.

Como esto es un sistema que involucra directamente a las personas, se debe ante todo prestar la mayor seguridad posible para que con ello no existan problemas que involucren a la integridad del personal que labora en el taller de los ferrocarriles matriz Riobamba.

\section{Fundamento Teórico}

\section{Elevador electromecánico}

Es un mecanismo empleado para la elevación de cargas pesadas que se ayuda de la combinación mecánica y electrónica para cumplir con su función. Su finalidad principal es ascender y descender un vehículo para la realización de su respectivo mantenimiento. (Ravaglioli S.p.A, 2018)

\section{Ensayos no destructivos}

Los ensayos no destructivos (NDT, por sus siglas en inglés) se efectúan de manera que la integridad del producto y la textura superficial permanezcan sin cambios. Estas técnicas suelen requerir una habilidad considerable del operario y tal vez sea difícil interpretar los resultados de las pruebas con precisión, debido a que las observaciones pueden ser subjetivas. Sin embargo, el uso de graficas de computadora y otras técnicas de mejoramiento ha reducido de manera considerable la posibilidad de error humano. (KALPAKJIAN, 2008)

Los ensayos no destructivos no ocasionan daño a los materiales ensayados, por lo cual después del examen dichos materiales pueden ser utilizados. (Pazos Peinado, 2006)

Estas pruebas permiten la identificación de materiales defectuosos antes de que sean confortados, maquinados ensamblados o puestos en servicio. El recazo o aceptación de una pieza con defectos depende del uso del componente. (Pazos Peinado, 2006) 
Las pruebas también se utilizan para la inspección periódica de componentes en servicio. La ocurrencia de una falla muchas veces puede ser evitada con el uso de estos ensayos. (Pazos Peinado, 2006)

\section{Tipos de ensayos no destructivos}

\section{Inspección visual}

La inspección visual es el método de END más común y más básico. Es de aplicación a una gran variedad de tipos de materiales y productos. Las posibilidades de detección de esta técnica se limitan, obviamente, a aquellos defectos que son visibles, tales como grietas, poros, desgaste, cavitación, decoloraciones, corrosión, etc., así como al control dimensional. (Gómez de León)

\section{Líquidos penetrantes}

Este método se puede utilizar para detectar varios defectos superficiales. El equipo es simple y de uso sencillo, puede utilizar para detectar varios defectos superficiales. Puede ser portátil y su operación es menos costosa que la de otros métodos. Sin embargo, solo puede detectar defectos que se encuentran abiertos a la superficie o que son externos. (KALPAKJIAN, 2008)

En esta técnica se aplican fluidos a las superficies de la parte para que penetren en grietas, costuras y poros. Por la acción de capilaridad, el penetrante puede introducirse en grietas has $0.1 \mu \mathrm{m}$ de anchura (KALPAKJIAN, 2008).

\section{Ultrasonido}

Optimiza el efecto de resonancia de las ondas para conseguir mediciones precisas de espesor de pared en tuberías, tanques, etc. Permite la detección de pérdidas de espesor producidas por fenómenos degradatorios como erosión, corrosión, Flow Acelerated Corrosion (FAC), etc. (Intertek Group plc, 2018)

\section{Ensayos para reconocimiento de material}

\section{Espectrometría}

La espectrometría es la técnica espectroscópica para tasar la concentración o la cantidad de especies determinadas. En estos casos, el instrumento que realiza tales medidas es un espectrómetro o espectrógrafo. La espectrometría a menudo se usa en física y química analítica para la identificación de sustancias mediante el espectro emitido o absorbido por las mismas. (Pérez, 2017) 


\section{Dibujo asistido por computadora CAD}

El acrónimo CAD (computer aided desingn) o en español; diseño asistido por ordenador, Se impuso desde que el desarrollo de los microprocesadores hizo posible crear, modificar y manipular gráficos complejos editados sobre la pantalla de un VDU (unidad de edición visual). (Hawkes, 1989)

CAD significa proceso de diseño que emplea sofisticadas técnicas graficas de ordenador. Apoyadas en paquetes de software para ayuda en los problemas analíticos, de desarrollo, de coste y ergonómicos asociados con el trabajo de diseño. (Hawkes, 1989)

\section{Metodología}

\section{Diseño de la investigación}

Se utiliza un diseño de investigación del tipo no experimental, siendo esta sistemática y empírica, se observará los resultados de los ensayos y análisis sobre los elevadores para describirlos y posteriormente tomar decisiones para su rehabilitación.

\section{Tipo de estudio}

El tipo de estudio que se realiza es del tipo descriptivo ya que se pretende establecer la situación de los elevadores electromecánicos antes de que tome lugar su rehabilitación mediante los ensayos de análisis no destructivos y el análisis de esfuerzos realizado sobre una digitalización en CAD.

\section{Métodos}

El método que se utiliza es el método deductivo, se partió de premisas generales en relación a las técnicas de END para sacar conclusiones de un caso particular, en este caso se busca explicar el estado de los elevadores electromecánicos y poniendo énfasis en la teoría para posteriormente determinar las acciones de rehabilitación.

El método analítico también es utilizado, se busca revisar y analizar ordenadamente los elementos o partes de los elevadores electromecánicos para determinar su situación y examinar las relaciones entre éstas.

\section{Técnicas e instrumentos}

En el estudio se plantea utilizar los tres tipos de ensayos no destructivos, inspección visual, inspección con tintas penetrantes y el análisis por ultrasonido, esto debido a que se tenía acceso a ellos gracias al laboratorio de resistencia de materiales de la Escuela Superior Politécnica de Chimborazo (ESPOCH). 
Se utiliza además la digitalización de los elevadores en CAD para correr un análisis de esfuerzos y lograr determinar el factor de seguridad y la carga máxima que pueden soportar, para ello también se utiliza la técnica de espectrometría de materiales para determinar los materiales a utilizar en la digitalización.

Finalmente se hace uso de una matriz de valoración la cual contribuirá a la decisión de las actividades de rehabilitación de los elevadores.

\section{Descripción del proceso}

Figura 1 se muestra en detalle el procedimiento a seguir para la ejecución el diagnóstico y decisión de actividades para la rehabilitación.

Figura 1. Procedimiento a seguir para la rehabilitación.

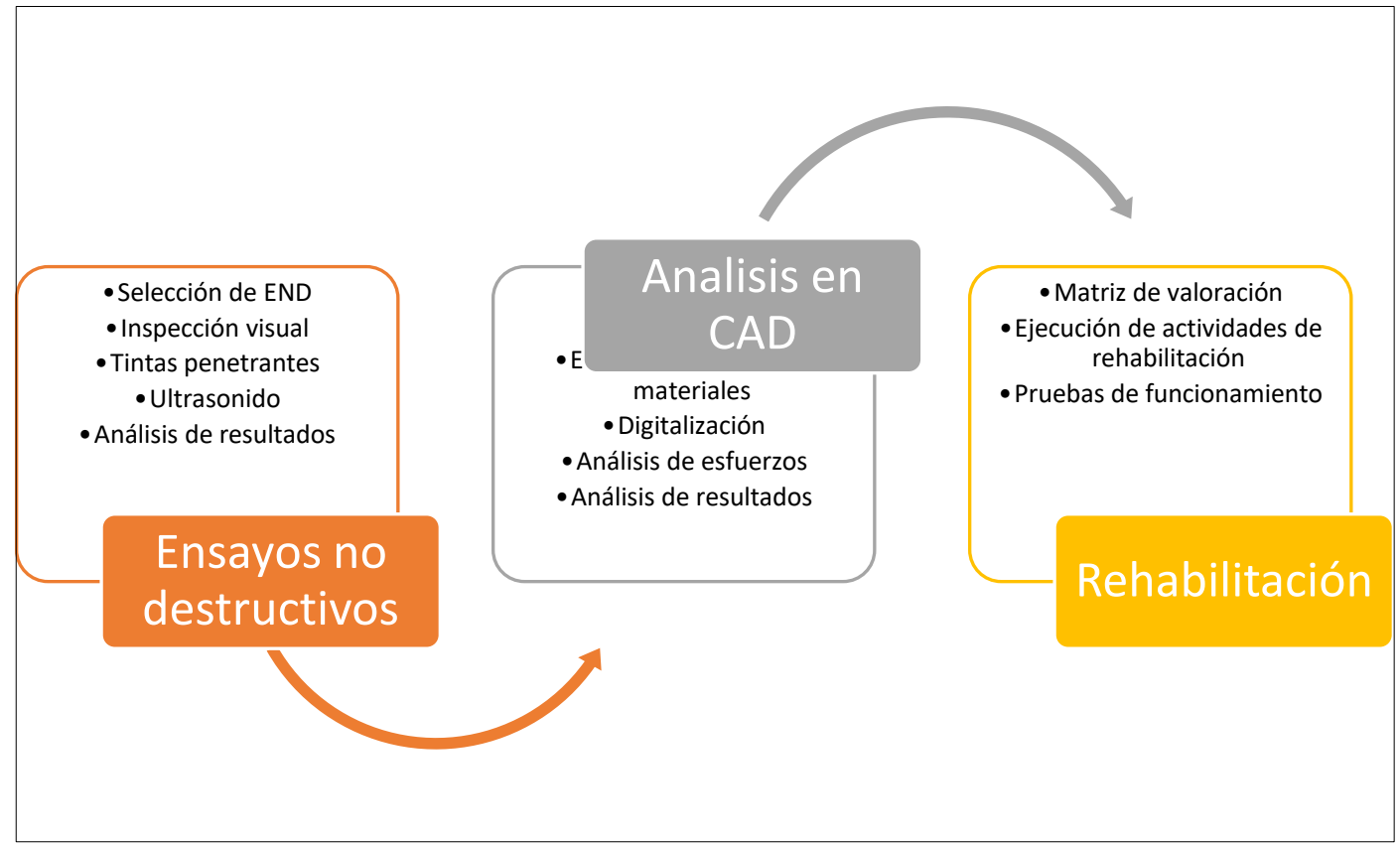

Elaborado por: Grupo de Investigación.

\section{Resultados}

El elevador electromecánico que se analiza y se muestra en la Figura 2 está constituido de una parte mecánica en la que se encuentran:

- Bastidor guía

- Husillo

- Ruedas retractiles

- Ruedas guías superiores e inferiores

- Brazo de carga

- Carro porta brazo 
- Desplazamiento manual del brazo

- Soporte de engranajes y motor eléctrico

- Depósito de grasa

- Engranaje recto principal y secundario

- Tornillo sin fin

- Pasador de soporte

- Engranaje helicoidal con engranaje recto y ejec de soporte

- Chumaceras

- Juntas del eje de soporte

- Palanca del eje de soporte

- Y una parte eléctrica constituida por:

- Motor eléctrico

- Tablero de mando principal

- Cajas de mando secundarios

- Enchufes y conexiones

Se comienza realizando los ensayos no destructivos y se tienen los siguientes resultados.

\section{Inspección Visual}

En este método el protagonista es el ojo humano que ayuda a la identificación de discontinuidades en la soldadura del elevador electromecánico, si es necesario se utiliza dispositivos ópticos que faciliten a la visualización de fallas en los cordones de suelda.

En primera instancia se cepillaron y limpiaron todas las áreas de la estructura a ser inspeccionadas, ya que la presencia de pintura de la soldadura dificultaba la inspección.

Figura 2. Elevador electromecánico

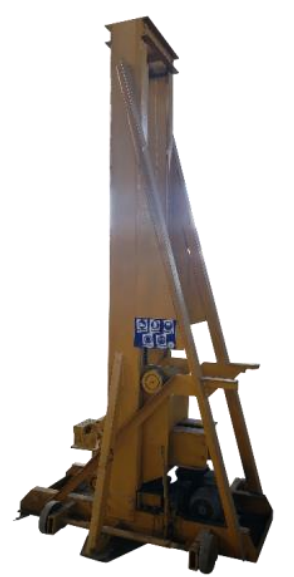


Con los cordones de soldadura listos para el reconocimiento de discontinuidades en caso de que estas existan, se procedió a la inspección para llegar a una conclusión con la evaluación realizada.

Se recomienda una distancia de $150 \mathrm{~mm}$ a $600 \mathrm{~mm}$ desde el ojo hasta la superficie a inspeccionar con un grado de inclinación de $30^{\circ}$, siendo esta una inspección visual directa tal como se indica en la figura.

Figura 3. Requisitos de distancia e inclinación para la inspección visual

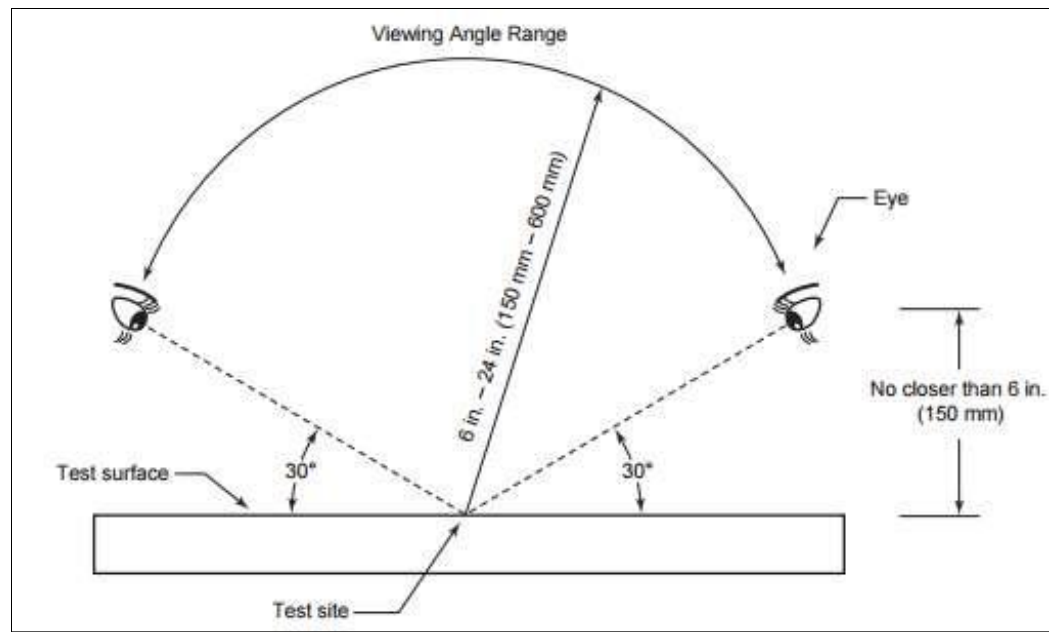

Como resultado se obtiene que existen geometrías variables en los cordones de soldadura, debido probablemente a un avance lento o rápido en la realización del cordón o el empleo de electrodos gruesos o delgados para la ejecución de la soldadura, esto es comprensible ya que no todas las zonas de unión serán sometidas a esfuerzos iguales, determinando que la soldadura tiene una valoración aceptable por lo cual se determina que los cordones de soldadura están bien ejecutados.

\section{Inspección por líquidos penetrantes}

Este método tiene una gran sensibilidad a la identificación de fisuras, discontinuidades, porosidad, etc. Para su aplicación se tomó en consideración la norma ASTM E 165 la cual indica el proceso (Figura 4) que se debe seguir para la aplicación de la inspección.

Después de realizar el proceso completo para la aplicación de tintas penetrantes de acuerdo a la norma, se pudo observar que tanto porosidades como fisuras no existen en el cordón seleccionado para efectuar el ensayo, sin embargo, la presencia de grietas en el borde del cordón de soldadura es visible, pero en proporción inferior al permisible en soldaduras. 
Los resultados se obtienen de medir las grietas en las partes analizadas y compararlos con la Tabla 1 el cordón de soldadura inspeccionado tiene un ancho de $10 \mathrm{~mm}$ y la grieta con mayor longitud en dicho cordón es de $14 \mathrm{~mm}$, siendo menor a $30 \mathrm{~mm}$ que sería el límite para que la soldadura apruebe el ensayo. En conclusión, la técnica de soldadura empleada para la unión de los elementos que conforman los elevadores tiene aceptabilidad.

Tabla 1. Indicación y proporciones para hallazgo de fallas

\begin{tabular}{ll}
\hline \multicolumn{1}{c}{ Indicaciones } & \multicolumn{1}{c}{ Proporción } \\
\hline Relevantes & Dimensión mayor a $1.5 \mathrm{~mm}$ \\
Lineales & $\begin{array}{l}\text { Longitud mayor a } 3 \text { veces su ancho } \\
\text { Redondeadas }\end{array}$ \\
& $\begin{array}{l}\text { Longitud igual o menor a 3 veces su } \\
\text { ancho }\end{array}$ \\
\hline
\end{tabular}

Elaborado por: Grupo de Investigación.

Figura 4. Proceso para la inspección por tintas penetrantes

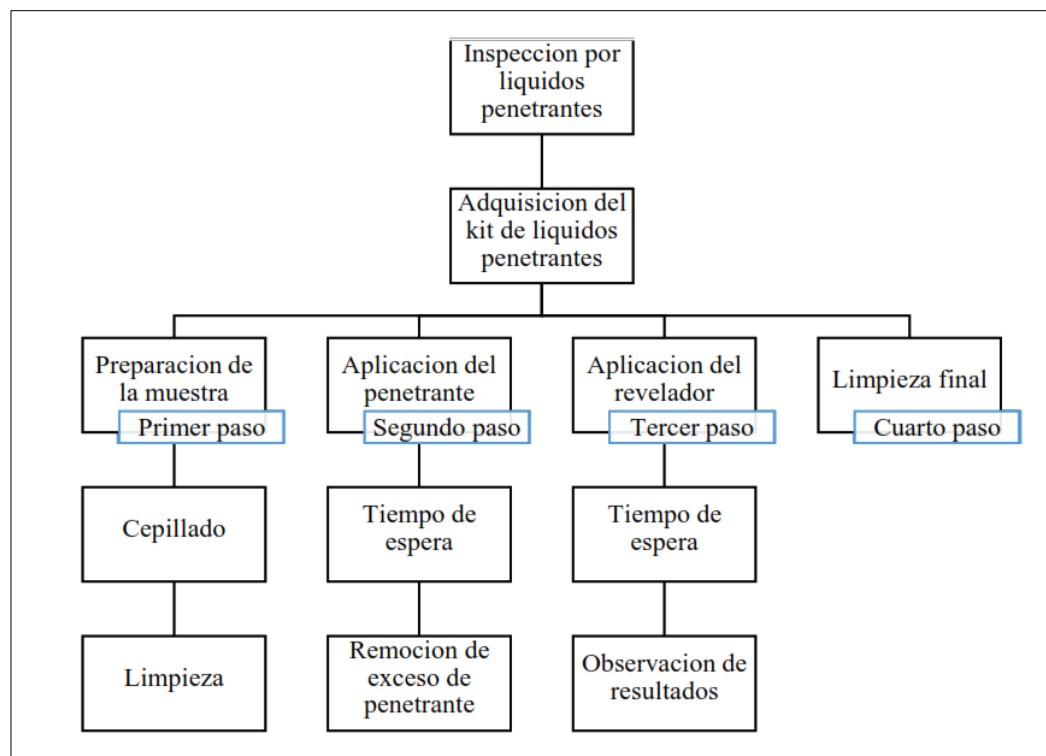

\section{Inspección por ultrasonido}

Como primer punto se calibra el medidor de ultrasonido, en este caso se utiliza un equipo Huatec FD 201B. Con un bloque de calibración establecemos el rango en el medidor y el material de la pieza con la que calibraremos el equipo. Se procede a la medición de ultrasonido en torno al cordón de soldadura con el transductor alrededor de la suelda. 
Para la interpretación de resultados se considera que la prueba se realizó en una calidad superficial rugosa por lo cual la ganancia del eco se mantendrá en un rango de $50 \%$ como máximo y un $0 \%$ como mínimo.

Al realizar la prueba de escaneo por ultrasonido se observó en la pantalla que después de que el transductor se haya desplazado en toda el área de barrido de la suelda, el eco de ultrasonido no excedía el $50 \%$ establecido, por lo tanto, el cordón de soldadura inspeccionado no tenía discontinuidades a tomar en consideración o de mayor relevancia.

\section{Digitalización y análisis de esfuerzos en los elevadores}

\section{Ensayo de espectrometría}

El tipo de espectrometría que se utiliza para la identificación del material es la espectrometría de plasma de corriente continua (DCP) en este caso el que se usa es de la marca Bruker. La prueba se realiza en muestras pertenecientes a un piñón del universo de engranajes, y una porción de la estructura del bastidor guía. Los resultados se muestran en la Tabla 2.

Tabla 2. Resultados del ensayo de espectrometría

\begin{tabular}{lll}
\hline Parte & Material & Módulo de Young \\
\hline Estructura del bastidor & Acero AISI 1020 & $209 \mathrm{GPa}$ \\
Tornillo sin fin & Acero AISI 1045 & $211 \mathrm{GPa}$ \\
\hline
\end{tabular}

Elaborado por: Grupo de Investigación.

Una vez determinados los materiales de los cuales están construidos los elevadores y realizadas las respectivas medidas y cálculos para la construcción de los engranajes, se procedió a la realización del CAD en el software Solid Works (Figura 2) del cual la ESPOCH facilitó su utilización por medio de las licencias respectivas.

\section{Análisis y simulación de esfuerzos en CAD}

Se procede a realizar un análisis estático de esfuerzos en Ansys, aplicando diferentes cargas en el lugar más crítico del elevador electromecánico el cual es el brazo de carga para evaluar la resistencia optima que puede soportar el elevador electromecánico.

\section{Análisis estático en el brazo.}

Para analizar la resistencia que el bastidor guía conjuntamente con el carro porta brazo puede soportar, se utiliza el software ANSYS, con ello se somete a diferentes cargas al elevador electromecánico, centrándose principalmente en el brazo de carga que es el lugar más crítico. 


\section{Mallado}

Se utiliza el mallado triangular, calidad del elemento (Element quality) para las diferentes cargas aplicadas en la estructura, se escogió este tipo de mallado porque la estructura de diseño tiene curvaturas y el mallado Element quality posee contornos de borde pequeños que se adaptan al diseño del elevador electromecánico, mejorando la calidad en toda la estructura. Posteriormente se corre un análisis de esfuerzos y deformaciones con una iteración de cargas de 4.25, 10, 13.9 y 15 T.

Figura 5. Fuerza actuante en la estructura

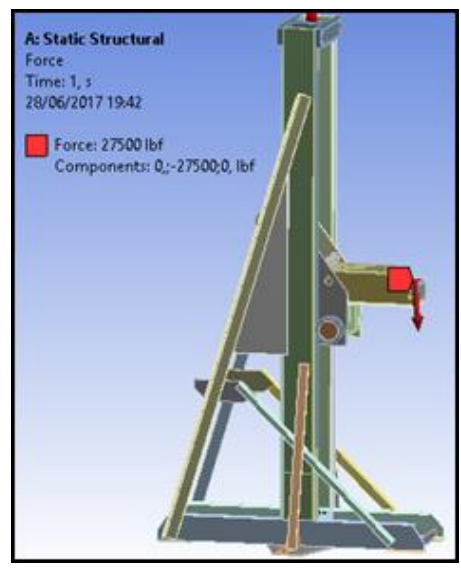

\section{Factor de seguridad}

En el estudio realizado podemos evidenciar que, al momento de aplicar una carga de 15 toneladas, el factor de seguridad tiene un valor de 1.05 el cual está en el límite del rango permisible, demostrando que dicha carga está dentro del régimen de seguridad y que a la larga provocará un déficit en la resistencia de la estructura. Los resultados en cuanto a el peso utilizado, esfuerzos, deformación se encuentran en la Tabla 3.

De acuerdo con los resultados del análisis de esfuerzos, las deformación tienden a elevarse cuando el brazo del elevador electromecánico está sometido a mayores cargas, con la entrevista realizada al jefe de taller de la empresa pública Ferrocarriles del Ecuador filial surestación Riobamba las cargas que experimentaran los elevadores electromecánicos tienen factores de seguridad que garantizan la utilización confortable de los mismos pero, si se aplica una carga de 15 toneladas al brazo de soporte, su factor de seguridad será de 1,05 que está al límite de lo permitido, recomendándose que en este caso es factible la incorporación de 2 elevadores adicionales para que su factor de seguridad garantice seguridad.

Tabla 3. Comparación del análisis estático del elevador 


\begin{tabular}{llllll}
\hline Carga & Malla & Esfuerzo & Deformación & $\begin{array}{l}\text { Factor de } \\
\text { Seguridad }\end{array}$ & Decisión \\
\hline $13,9 \mathrm{~T}$ & $\begin{array}{l}\text { Element } \\
\text { quality }\end{array}$ & 0,0012 & $0,148 \mathrm{~cm}$ & 1,13 & Adecuado \\
$15 \mathrm{~T}$ & $\begin{array}{l}\text { Element } \\
\text { quality }\end{array}$ & 0,0013 & $0,160 \mathrm{~cm}$ & 1,05 & Adecuado \\
$4,25 \mathrm{~T}$ & $\begin{array}{l}\text { Element } \\
\text { quality }\end{array}$ & 0,0003 & $0,045 \mathrm{~cm}$ & 3,7 & Adecuado \\
$10 \mathrm{~T}$ & $\begin{array}{l}\text { Element } \\
\text { quality }\end{array}$ & 0,0008 & $0,106 \mathrm{~cm}$ & 1,57 & Adecuado \\
\end{tabular}

Elaborado por: Grupo de Investigación.

\section{Discusión}

Luego de exponer los resultados se procede a discutir los resultados y diagnosticar estructuralmente a los elevadores de lo cual se desprende que de la realización de los END en este caso aplicados a la estructura y con énfasis en los cordones de soldadura han demostrado ser técnicas eficaces al momento de verificar la construcción de este tipo de maquinaria. Gracias a estas técnicas se puede validar de que a pesar de encontrarse irregularidades en los cordones de suelda estos todavía podían ser considerados como permisibles al momento de su utilización tal como se presentó en los resultados.

Con respecto a la digitalización, para que esta pueda ser llevada a cabo era necesario la determinación del material del que estaban construidos los elevadores por lo cual la técnica de espectrometría fue de gran utilidad al permitir determinar el tipo de material y con ello asegurar la eficacia de la digitalización.

En el software Ansys se pudo simular las cargas a las cuales van a estar sometidos estos elevadores, y considerando la calidad del mallado y los resultados, luego del análisis de estas pruebas se le puede presentar a la empresa los esfuerzos máximos a los cuales pueden operar, es el caso de la Tabla 3.

En el caso de las deformaciones y esfuerzos la zona más crítica se demuestra está en el brazo del elevador electromecánico, y en la simulación al someterlo a diversos tipos de carga se pudo corroborar que toda la estructura demostró el no presentar una excesiva deformación (Figura 6) y estar acorde para poder elevar los pesos de los vehículos ferroviarios con los que cuenta la empresa que en este caso pueden presentar pesos desde las 13 toneladas hasta las 66. Acotando que de acuerdo a los cálculos es recomendado el que cada elevador con el 
que se cuente puede trabajar con hasta $15 \mathrm{~T}$, pero con la recomendación de que se utilicen dos elevadores para manejar ese peso.

En el caso de la locomotora Alsthon que pesa 55.665 toneladas se recomienda se distribuya el peso para 4 elevadores que de acuerdo a los estudios realizados (Tabla 3) estarían soportando un peso de 13.9 $\mathrm{T}$ y manejando un factor de seguridad de 1.13.

Figura 6. Esfuerzos con una carga de $15 \mathrm{~T}$

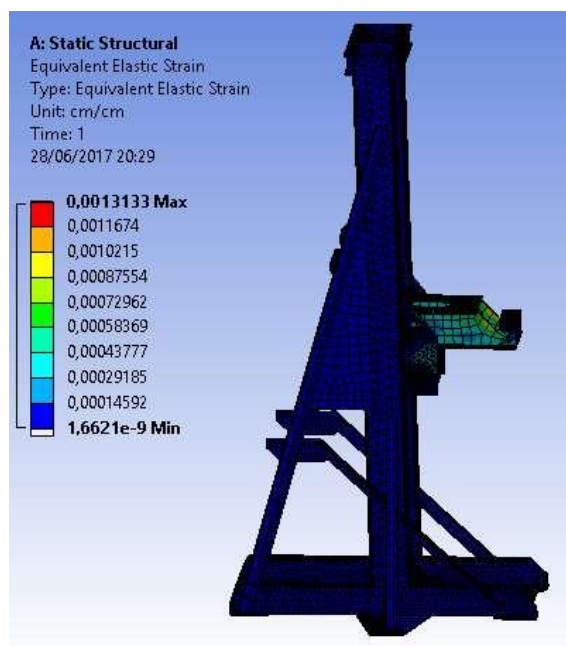

Elaborado por: Grupo de Investigación.

Figura 7. Deformación con carga de $15 \mathrm{~T}$

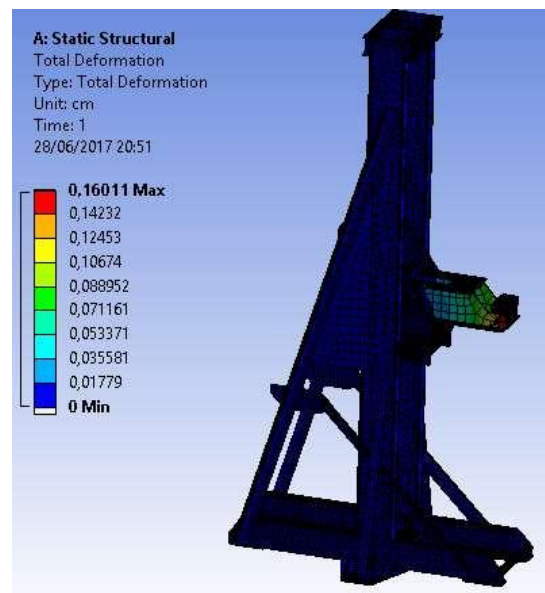

Elaborado por: Grupo de Investigación.

La determinación del factor de seguridad permite el dar a conocer a la empresa FEEP, el que el diseño de las máquinas a rehabilitar y posteriormente usar en el servicio de mantenimiento, pueden brindarles la seguridad necesaria en cuanto se refiere al diseño.

La consecución de todos los tipos de ensayo y el asegurar que el diseño y que las partes de soldadura y construcción son adecuadas para poder comenzar una rehabilitación se procede 
a aplicar una matriz de valoración en la cual se puede identificar los trabajos de rehabilitación a ejecutarse. En la tabla se muestra la matriz de valoración aplicada a la estructura, se ejecutan matrices para la valoración del conjunto de engranajes, medios de sujeción, sistema eléctrico, señalización de seguridad, y mediante sus resultados se establecen las acciones de mejora.

Se realiza acciones de rehabilitación en todos los componentes de cuatro elevadores diagnosticados, y se consigue su rehabilitación y puesta en marcha, terminándose el trabajo con una prueba en la que se evidencia la confiabilidad en cuanto al funcionamiento y a la veracidad de los resultados obtenidos en cada uno de los ensayos ejecutados.

Tabla 4. Extracto de la matriz de valoración de la estructura.

\begin{tabular}{|c|c|c|c|c|}
\hline Elemento & $\begin{array}{l}\text { Muy } \\
\text { bueno }\end{array}$ & Bueno & Regular Malo & $\begin{array}{ll}\text { Acciones } & \mathrm{de} \\
\text { rehabilitación } & \end{array}$ \\
\hline Bastidor guía & & $\mathrm{X}$ & & $\begin{array}{l}\text { Remoción de pintura, } \\
\text { limpiar, lijar y pintar. }\end{array}$ \\
\hline Brazo de carga & & $\mathrm{X}$ & & $\begin{array}{l}\text { Remoción de pintura, } \\
\text { limpiar, lijar y pintar. }\end{array}$ \\
\hline Carro porta brazo & & $X$ & & $\begin{array}{l}\text { Remoción de pintura, } \\
\text { limpiar, lijar y pintar. }\end{array}$ \\
\hline $\begin{array}{l}\text { Desplazamiento } \\
\text { manual del brazo }\end{array}$ & & $\mathrm{X}$ & & $\begin{array}{l}\text { Remoción de pintura, } \\
\text { limpiar, lijar y pintar. }\end{array}$ \\
\hline $\begin{array}{l}\text { Soportes del eje de } \\
\text { tornillo sin fin }\end{array}$ & & & $\mathrm{X}$ & $\begin{array}{l}\text { Remoción de pintura, } \\
\text { limpiar, reparar } \\
\text { pintar }\end{array}$ \\
\hline
\end{tabular}

Elaborado por: Grupo de Investigación.

La prueba consistió en elevar un vagón panorámico de 17 T de peso aproximadamente según los datos técnicos, los elevadores se ubicaron en la parte media de cada boggie para equilibrar las cargas a las que se someterán dos de los cuatro elevadores rehabilitados.

Los resultados fueron satisfactorios ya que se consiguió elevar este vagón a $1 \mathrm{~m}$ sobre el suelo en $1.28 \mathrm{~s}$, sin presentar novedades en cuanto a la integridad de la estructura, demostrándose la que el diseño y la construcción son adecuadas a los requisitos de operación y de que las acciones de rehabilitación fueron eficaces.

Figura 8. Prueba de elevación de vagón panorámico. 


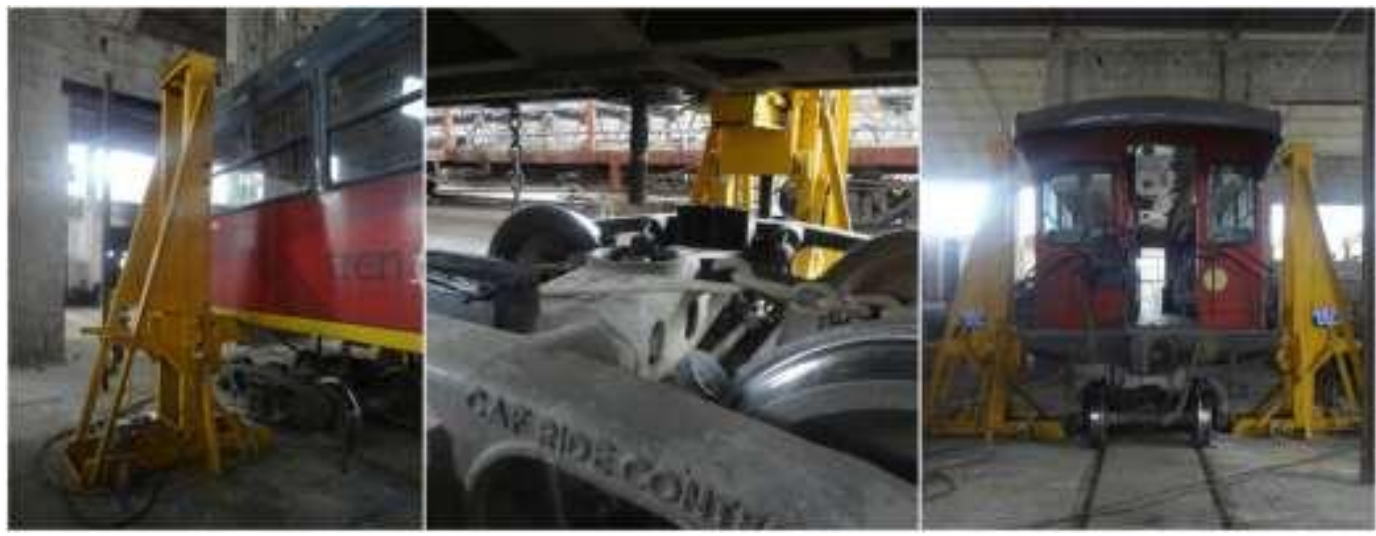

Elaborado por: Grupo de Investigación.

Uno de los propósitos de este estudio también estaba en el demostrar que la utilización de la metodología propuesta y que se expresa en el procedimiento de la Figura 1, resulta ser adecuado al momento de rehabilitar este tipo de maquinarias y que además proporciona las evidencias suficientes para validar un diseño y una construcción al momento de proceder con un trabajo de rehabilitación.

\section{Conclusiones.}

- Se utilizó métodos de ensayos no destructivos convenientes para diagnosticar la soldadura de la estructura de los elevadores electromecánicos, en este caso la aplicación de líquidos penetrantes y ultrasonido asistieron para la posterior aprobación de los cordones de soldadura.

- Se analizó en el software ANSYS y SOLIDWORKS los esfuerzos a los que se ven sometidos los elevadores electromecánicos y determinar su admisibilidad a través de los resultados del factor de seguridad admisible que se obtuvo, utilizando las diferentes cargas que poseen los vagones ferroviarios y así proporcionar con los resultados obtenidos mayor seguridad y funcionabilidad de trabajo en la estructura.

- Se revisó cada uno de los elementos que conforman los elevadores electromecánicos con su respectiva funcionalidad, para elaborar una matriz de valoración con la asistencia de una adecuada inspección visual y ayuda proporcionada por los resultados de los ensayos no destructivos, para analizar la situación actual en la que se encontraban los elevadores electromecánicos.

- Con una carga de 15 toneladas por elevador electromecánico, su factor de seguridad es de 1,05 estando al margen de lo permitido y cuyo peso es el máximo que puede soportar cada elevador electromecánico por razones de infalibilidad.

- Se rehabilitó los elevadores electromecánicos una vez validadas las pruebas y ensayos a los elementos que los conforman, y ya montado el universo de engranajes se verificó el correcto funcionamiento de todo el mecanismo, para su posterior empleo en el campo laboral. 


\section{Referencias bibliográficas.}

AEND, (. e. (2009). Ultrasonidos: nivel II. (P. E. Central, Ed.) FC Editorial, 2009. Obtenido de https://ebookcentral.proquest.com/lib/espochsp/detail.action?docID=4849810.

AEND, (. e. (s.f.). Líquidos penetrantes: nivel I. (P. E. Central, Ed.) FC Editorial, 2009. Obtenido https://ebookcentral.proquest.com/lib/espochsp/detail.action?docID=4849806.

American Petroleum Institute. (2004). Welding Inspection and Metallurgy. API Publishing Services. Obtenido de http://lecturer.ppns.ac.id/munir/wpcontent/uploads/sites/14/2015/09/41099933-Welding-Inspection-andMetallurgy.pdf

ASTM. (2017). Norma ASTM E165 / E165M - 12. Obtenido de https://www.astm.org/Standards/E165.htm

Delojo, M. G. (2011). Inspección visual: niveles II y III. (P. E. Central, Ed.) FC Editorial. Obtenido de https://ebookcentral.proquest.com/lib/espochsp/detail.action?docID=4849805.

Gómez de León, F. (s.f.). Universidad de Murcia. Recuperado el 16 de mayo de 2017, de http://ocw.um.es/ciencias/resistencia-de-materiales-y-calculo-deestructuras/material-de-clase-1/tema07.pdf

Hawkes, B. (1989). CADCAM. Madrid: Paraninfo.

Hristoforou, E. (2018). Advanced Non-Destructive Testing in Steels. 492.

Intertek Group plc. (2018). intertek.es. Obtenido de http://www.intertek.es/

KALPAKJIAN. (2008). Manufactura, ingeniería y tecnología. Mexico: Person education.

Mohammad Yousefieh, M. S. (2017). Ensayos no destructivos de soldaduras. IUT. doi:978600-8157-25-0

Pazos Peinado, N. (2006). Tecnología de los metales y procesos de manufactura. Caracas: Publicaciones UCAB.

Pérez, G. (2017). ESPECTROMETRIA.COM. Obtenido de https://www.espectrometria.com Ravaglioli S.p.A. (2018). Ravaglioli. Obtenido de https://ravaglioli.com/

Viswanathan, K. (2018). Non-destructive testing methods and their Applications. UBT International Conference, 391-417. doi:10.33107/ubt-ic.2018.182 
Para citar el artículo indexado.

Buenaño L., Castillo E., Cuaical B. \& Padilla C. (2019). Ensayos no destructivos y rehabilitación de elevadores de vehículos ferroviarios. Revista electrónica Ciencia Digital 3(1), 333-350. Recuperado desde: http://cienciadigital.org/revistacienciadigital2/index.php/CienciaDigital/article/view/295/70 $\underline{3}$

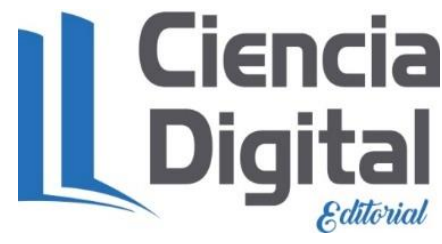

El artículo que se publica es de exclusiva responsabilidad de los autores y no necesariamente reflejan el pensamiento de la Revista Ciencia Digital.

El artículo queda en propiedad de la revista y, por tanto, su publicación parcial y/o total en otro medio tiene que ser autorizado por el director de la Revista Ciencia Digital.
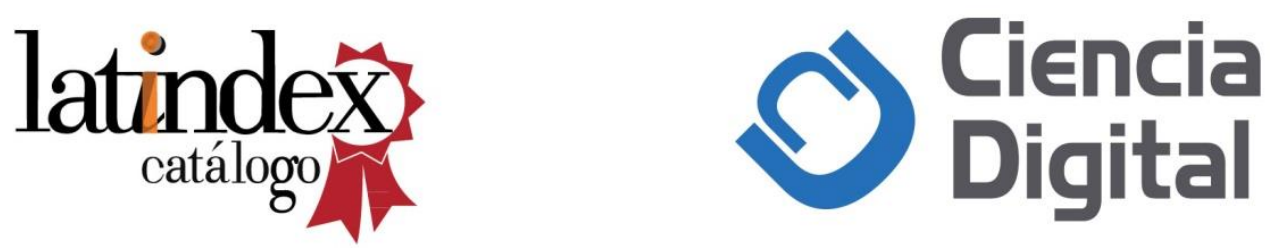